\title{
Deployment and demonstration of wide area monitoring system in power system of Great Britain
}

\author{
Peter WALL ${ }^{1}$, Papiya DATTARAY ${ }^{1}$, Zhaoyang JIN ${ }^{1}$, \\ Priyanka MOHAPATRA ${ }^{2}$, James YU $^{2}$, Douglas WILSON ${ }^{3}$, \\ Karine HAY ${ }^{3}$, Stuart CLARK ${ }^{3}$, Mark OSBORNE ${ }^{4}$, Phillip M. ASHTON ${ }^{4}$, \\ Vladimir TERZIJA ${ }^{1}$
}

\begin{abstract}
The creation of a suitable wide area monitoring system (WAMS) is widely recognized as an essential aspect of delivering a power system that will be secure, efficient and sustainable for the foreseeable future. In Great Britain (GB), the deployment of the first WAMS to monitor the entire power system in real time was the responsibility of the visualization of real time system dynamics using enhanced monitoring (VISOR) project. The core scope of the VISOR project is to deploy this WAMS and demonstrate how WAMS applications can in the near term provide system operators and planners with clear, actionable information. This paper presents the wider scope of the VISOR project and the GB wide WAMS that has been deployed. Furthermore, the paper describes some of the WAMS applications that have been deployed and provides
\end{abstract}

CrossCheck date: 12 June 2016

Received: 23 March 2016/Accepted: 12 June 2016/Published online: 13 July 2016

(C) The Author(s) 2016. This article is published with open access at Springerlink.com

$\triangle$ Peter WALL

peter.wall@manchester.ac.uk

Papiya DATTARAY

papiya.dattaray@manchester.ac.uk

Zhaoyang JIN

zhaoyang.jin@manchester.ac.uk

Priyanka MOHAPATRA

pmohapatra@scottishPower.com

James YU

james.yu@scottishpower.com

Douglas WILSON

douglas.wilson@alstom.com

Karine HAY

karine.hay@alstom.com examples of the measurement device performance issues that have been encountered during the project.

Keywords Model validation, Line parameter estimation, Subsynchronous oscillation, Subsynchronous resonance synchronized measurement technology, Wide area monitoring

\section{Introduction}

Wide area monitoring systems (WAMSs) represent the future of power system monitoring [1,2] and several recent reports on wide area blackouts have stated that they may contribute to limiting the likelihood and severity of similar blackouts in the future $[3,4]$.

\section{Stuart CLARK}

stuart.clark@alstom.com

Mark OSBORNE

mark.Osborne@nationalgrid.com

Phillip M. ASHTON

phil.Ashton@nationalgrid.com

Vladimir TERZIJA

vladimir.terzija@manchester.ac.uk

Department of Electrical and Electronic Engineering, The University of Manchester, Manchester M13 9PL, UK

2 SP Energy Networks, Ochil House, Hamilton International Park, Blantyre G72 0HT, UK

GE Grid Solutions, 1 Tanfield, Edinburgh EH3 5DA, UK

4 National Grid, 1-3 Strand, London WC2N 5EH, UK 
A WAMS enables the real-time monitoring of power system dynamics by bringing together new developments in the fields of measurement, communication and computing [5]. Measurements of voltage and current phasors are recorded by phasor measurement units (PMUs) installed across a wide area power system and time tagged at the point of measurement using a common time reference (e.g. using GPS). Synchronizing the angle measurements to a common time reference allows them to be combined into a single data record in real time that represents a snap shot of the system at that time, which with existing technology is updated at a rate of up to once per cycle. With suitable supporting communication and computing resources a sequence of these snap shots can be used to visualize system dynamics in real time.

However, these synchronized snap shots can be used for far more than visualization of dynamics. Advances in the computing resources available to power system engineers have enabled the development of a wide range of new algorithms that process WAMS data online to support the operation of power systems. Examples of these online WAMS applications include the real time estimation of oscillation parameters (e.g. inter area oscillations), the dynamic rating of transmission lines, and hybrid and linear state estimation.

Furthermore, the synchronized nature of WAMS data means that it is ready for immediate use as part of offline applications (e.g. post mortem analysis of events and model validation) without the need for engineers to perform time consuming and error prone manual time alignment.

However, given the complexity of a WAMS and the significant capital and operational expenditure that will be associated with any large scale WAMS deployment, the cost effectiveness of WAMS is yet to be demonstrated sufficiently for business as usual deployment in GB.

The VISOR project is a GB innovation project led by SP Energy Networks (SPEN) that brings together the three GB transmission system owners (SPEN, National Grid and SSE), the GB system operator (National Grid), researchers (The University of Manchester) and vendors (GE Grid Solutions). The core goal of VISOR is to create the first WAMS that monitors the entire GB system and then to use this WAMS to showcase the tangible benefits of WAMS applications to GB system.

VISOR is an innovation project; in the context of GB this means that VISOR is funded using customer money that is released through the network innovation competition (NIC) [6]. Innovation funding allows the transmission owners/operator in GB to trial new technologies/arrangements that are not yet ready for business as usual deployment, without violating their obligation to provide a cost effective, high quality and secure supply of electricity.
Therefore, the motivation for VISOR project is to help build the case for business as usual deployment of WAMS in the GB system. An innovation project is necessary to achieve this because, whilst the many potential operational benefits of WAMS are well reported, the tangible business benefits of most WAMS applications are yet to be demonstrated and quantified in practice. More details about the VISOR project can be found in the initial project submission [7].

Furthermore, at this time, an accepted common WAMS architecture or standardized approach for developing a WAMS does not exist [8]. So, it is necessary to investigate how best to design and deploy a WAMS for GB system.

VISOR will focus on the role of WAMS in the following areas of power system monitoring, operation and planning.

1) Monitoring and alarming for subsynchronous oscillations (SSO) in the frequency range of $0.002-46 \mathrm{~Hz}$.

2) Localizing the source of SSO.

3) Dynamic model validation.

4) Reducing the impact of uncertainty on security limits.

5) Hybrid state estimation (HSE).

6) Line parameter estimation (LPE) using PMUs.

7) Optimal placement of synchronized measurement technology (SMT) for monitoring SSO.

8) Laboratory testing of SMT.

The paper is structured as follows. Section 2 introduces the GB power system. Section 3 presents the WAMS that is being deployed by the VISOR project and introduces the waveform measurement units (WMUs) that are being trialed for the first time as part of it. Section 4 describes some of the applications that are being studied as part of VISOR. Finally, Section 5 provides examples of some of the device performance issues that have been encountered during the project and Section 6 concludes the paper.

\section{GB power system}

The GB power system is an isolated power system with no synchronous connections to neighboring power systems, although it does have HVDC connections to the power systems in Northern Ireland (500 MW), the Republic of Ireland (500 MW), France (2000 MW), and the Netherlands $(1 \mathrm{GW})$. It is a winter peaking system with a nominal frequency of $50 \mathrm{~Hz}$, peak demand of approximately $53 \mathrm{GW}$ and installed generation capacity of $80 \mathrm{GW}$.

Like most systems in the world, the GB power system is experiencing a time of significant change, including [9]:

1) The closure of many large thermal generators.

2) The first installations of fixed and thyristor controlled series compensation (TCSC). 
3) Increasing HVDC interconnection to neighboring power systems.

4) The first installation of HVDC in parallel to the existing AC system.

5) Construction of large offshore wind farms.

6) Increasing penetration of distributed generation (particularly solar PV and wind).

7) New gas and nuclear power plants will be significantly larger in terms of MW rating than existing plant.

Many of these changes are driving a profound shift in the generation mix of the GB power system, which is raising concerns about the reduction in system inertia, variation in fault levels and the management of voltage [10]. An example of the impact these changes are already having on the GB power system is the increase in the size of the largest credible single loss of infeed from 1320 to $1800 \mathrm{MW}$.

The GB system contains a single transmission system operator (National Grid SO) and three different transmission owners (National Grid Electricity Transmission (NGET)—England and Wales, SPEN-South of Scotland, and SHE Transmission-North of Scotland). The power flow between these transmission owner areas can usually be characterized by a significant flow of power from low cost generation in Scotland to the load centers in England. The boundary between these areas is called Boundary 6 (B6) of the GB power system. The changing generation mix in GB (primarily the connection of significant quantities of wind generation in Scotland) will further increase the required power flow on this boundary if the load is to be served at minimum cost.

\subsection{Boundary 6}

The SPEN system is connected to the NGET system in the North of England via two double $400 \mathrm{kV}$ AC circuits. These circuits, referred to as the East and West Interconnections, along with some neighboring circuits at $132 \mathrm{kV}$, make up the Anglo-Scottish constraint boundary (B6). This boundary is shown in Fig. 1 and marked in the figure are the locations of the series compensation, both fixed and thyristor controlled, and intra-network HVDC link on the west coast that will be installed to enhance the B6 boundary.

The boundary is stability limited to around $2500 \mathrm{MW}$, requiring security under contingency of either the eastern or western interconnectors. The limit can be increased to approximately $3300 \mathrm{MW}$ with the arming of an operational tripping scheme (OTS). At day-ahead timescales the maximum capacity of the boundary is required to be planned such that the system does not experience any instability, unacceptable voltage condition, or overloading of any network assets for any credible system fault. The Security and Quality of Supply Standard (SQSS) defines

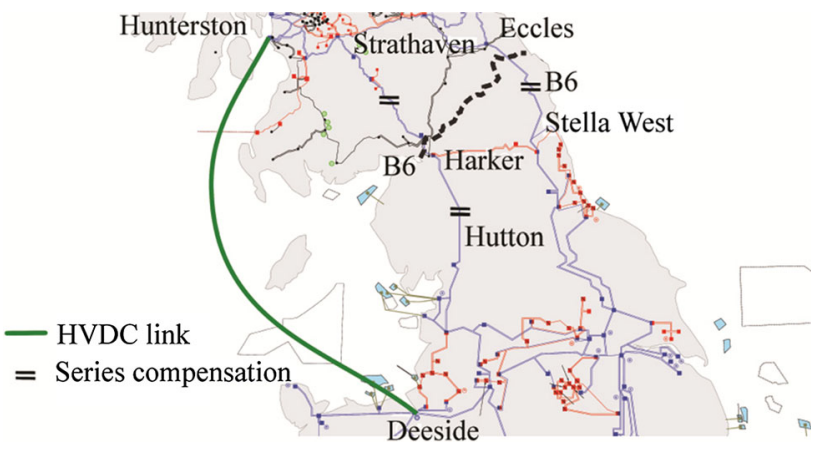

Fig. 1 B6 reinforcements

the limits for these conditions [11], specifically it defines system instability in terms of pole slipping and poor damping.

In order to accommodate the increasing volumes of wind power connecting to the Scottish network, a program of upgrades to the capability of B6 is in progress. The connection of TCSC, fixed series compensation (FSC) and intra-network HVDC links should see the limit increase to $6600 \mathrm{MW}$ by 2017 ; these enhancements are marked in Fig. 1. In line with National Grid's Gone Green 2014 scenario, the requirements of B6 could be greater than $11 \mathrm{GW}$ by 2035 [9].

With the continued schedule of changes to the GB transmission system from growing renewable deployment and increasing variability in power flows, a robust and ongoing system monitoring solution is required. The study of how WAMS can increase the secure power flow on this boundary is one of the key aspects of the VISOR project.

\section{VISOR WAMS}

The GB wide WAMS that VISOR is deploying builds upon previous WAMS deployments within SPEN and NGET with the goal of providing visibility of all three transmission owner (TO) areas to the GB SO.

A schematic of the VISOR WAMS is presented in Fig. 2, this shows how the existing WAMS assets in GB have been integrated into the VISOR WAMS.

Initially the communication between each of the three new TO level data centers and the SO level data hub will use an IPSec link. However, during the course of the project a MPLS link will be established between the SPEN data center and the data hub to accommodate the larger amount of data that will be streamed from this TO network.

\subsection{Waveform measurement unit}

The majority of the measurement devices in the VISOR WAMS will be PMUs. PMUs are the most widely used 


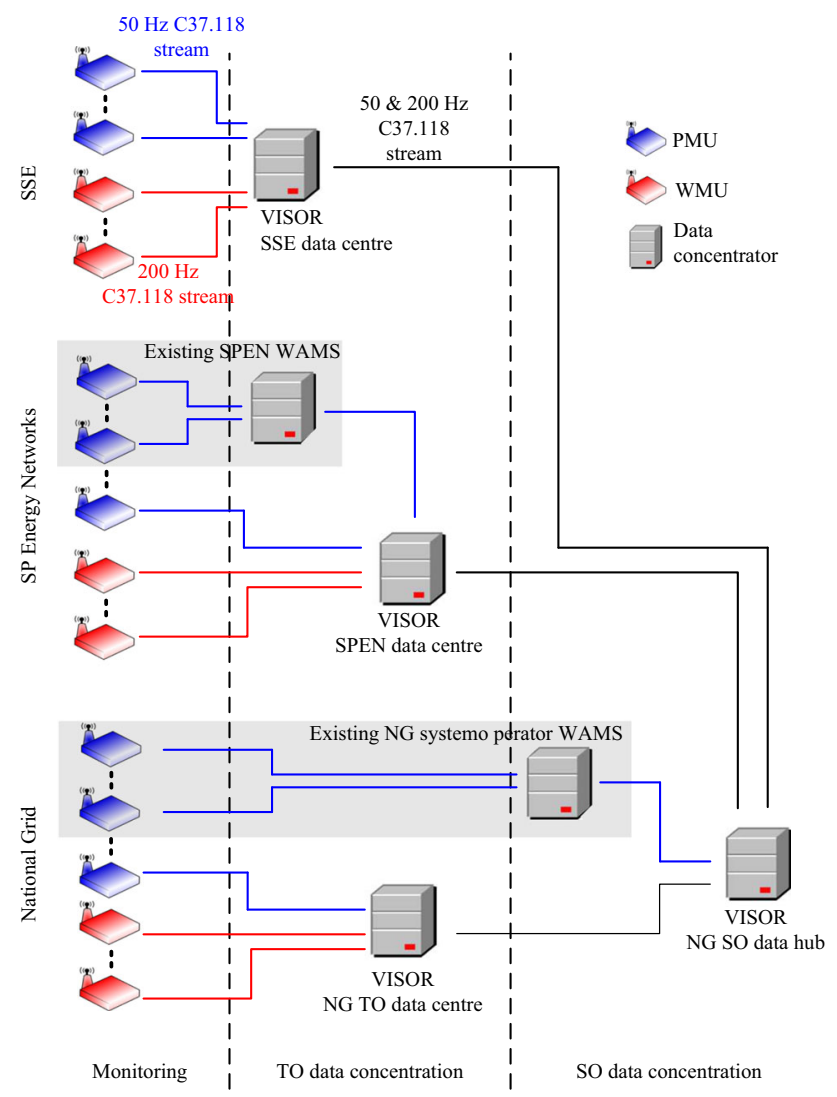

Fig. 2 Schematic of VISOR WAMS

synchronized measurement device [5]. However, an innovative feature of the VISOR WAMS is that it will include the first live trial of a $200 \mathrm{~Hz}$ WMU that is fully C37.118.2 compliant, which will be used to monitor SSO up to $46 \mathrm{~Hz}$ in real time. Once complete, the VISOR WAMS will receive real-time data streams from approximately 60 PMUs and 10 WMUs.

The WMU is a synchronized measurement device that provides point-on-wave measurements at a rate of $200 \mathrm{~Hz}$. For clarity, Fig. 3 provides a comparison of the output of a PMU and a WMU when exposed to a $10 \mathrm{~Hz}$ oscillation. These waveform measurements are then streamed using the analogue value data type that is defined in the IEEE C37.118.2 standard [12]. The trial of this device forms a key aspect of VISOR, as it can provide visibility of subsynchronous oscillations that cannot be accurately reported by $50 \mathrm{~Hz}$ PMUs, which are limited to $25 \mathrm{~Hz}$ by the Nyquist limit and to around $10-20 \mathrm{~Hz}$ by the length of window used for ensuring robust phasor calculation.

The number of measurement devices installed as part of VISOR had to be carefully considered. This was to ensure that the level of expenditure was appropriate. This was particularly challenging, as the exact number of PMUs/the PMU placements required for many of the considered applications has not, at this time, been defined. This means

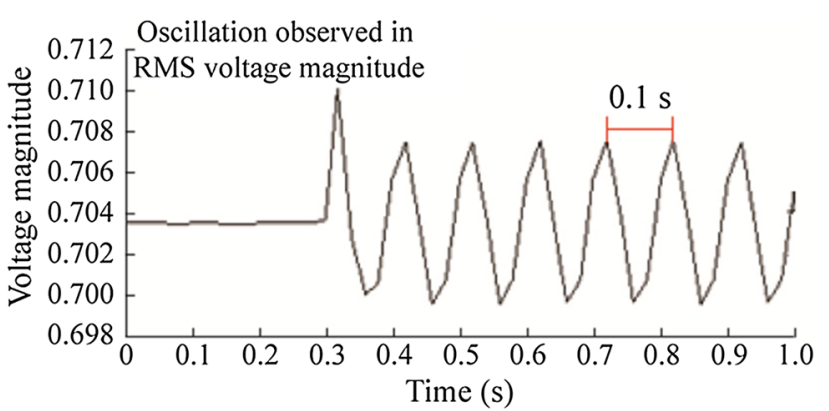

(a) PMU

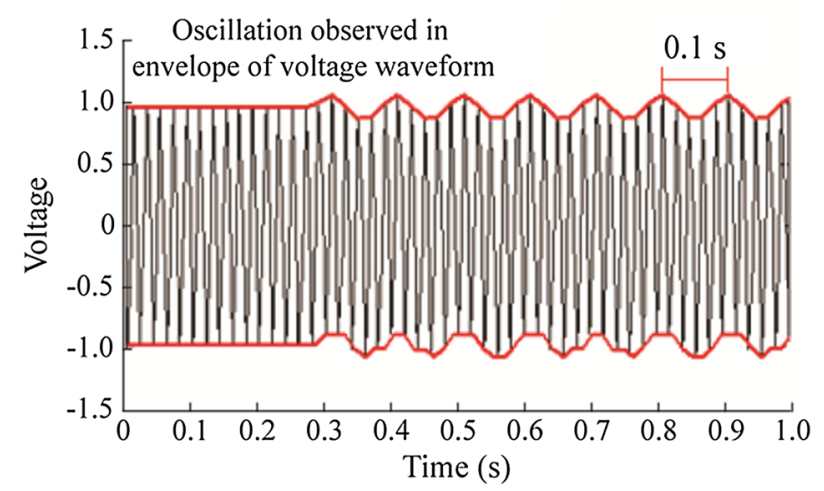

(b) WMU

Fig. 3 Simulated comparison of output of a PMU and a WMU when exposed to a $10 \mathrm{~Hz} \mathrm{SSO}$

that the number of PMUs installed may be insufficient to support some of the applications at the innovation level and/or the production level (i.e. as part of business as usual operation of the GB system). One of the key roles of VISOR is to assess the PMU support required for each application to provide a tangible benefit to the GB system. This understanding will then inform the creation of a roadmap for WAMS deployment in GB. The scale and complexity of a WAMS means that a proper roadmap is vital for their deployment [13, 14]. A roadmap will help to ensure that the WAMS is designed and built optimally (both in terms of expenditure and performance). Therefore, a roadmap of this nature is one of the essential outputs of VISOR, as it will help ensure that any further WAMS deployment in GB provides value for money to the consumer.

\section{VISOR applications}

The VISOR WAMS will be used to demonstrate the potential benefit that may be offered to the GB power system in the near term by a selection of WAMS applications. Many WAMS applications have been deployed in power systems and many more proposed, examples are listed in $[1,2,5]$. The applications studied in VISOR were 
selected from those that have reached a sufficient degree of maturity for use in the near term and based on the needs of the GB power system.

\subsection{Subsynchronous oscillation monitoring, visualization, source location and alarming}

The monitoring of oscillations has been broken down into three ranges as part of VISOR, these are:

1) Very low frequency (VLF): $0.002-0.1 \mathrm{~Hz}$.

2) Low frequency (LF): $0.1-4 \mathrm{~Hz}$.

3) Subsynchronous (SS): 4-46 Hz.

Applications are being deployed within the VISOR WAMS to deliver real time monitoring, visualization and alarming of all three ranges. Furthermore, a new application is being deployed that can, in real time, locate the source (or the measurement location closest to the source) of oscillations in the $0.002-4 \mathrm{~Hz}$ range.

\subsubsection{VLF monitoring}

The monitoring of the VLF range is of interest in the GB system due to the changes that are occurring in the generation mix and the increasing reduction and variation in system inertia.

Real time monitoring of the VLF range of oscillation is within the capabilities of the existing measurement technology. However, it has not been widely pursued to date because the parameter estimation methods deployed have had their performance tuned to the LF range, as this contains the inter area oscillations that have been the most relevant concern for most operators.

Simply extending the band over which the existing methods are expected to accurately estimate the parameters of any oscillation would require the use of a longer data window, to accurately capture the longer period of the VLF oscillations, which would delay detection of LF oscillations and limit the accuracy of the estimation of their parameters. Therefore, it is preferable to develop a separate monitoring approach to capture these oscillations. However, the concern over these VLF oscillations is not sufficient to justify the cost of this development as part of business as usual. Therefore, as an innovation project, VISOR is trialing the deployment of a dedicated VLF monitoring tool. By installing this monitoring now it may be possible to benchmark the VLF modes in the GB system and then study how they vary as the system evolves.

The VLF monitoring method processes measurements of frequency to estimate the amplitude and phase of the dominant oscillation in the VLF range. The common mode nature of VLF oscillations in power systems makes the phase difficult to estimate and success is dependent on having high resolution frequency measurements. Damping is not estimated, as the long periods of VLF oscillations makes it difficult to estimate accurately and of questionable value.

\subsubsection{SS monitoring}

The monitoring of SS oscillations in the range of $4-46 \mathrm{~Hz}$ is of interest in the GB power system due to the recent installation of the first FSC in the GB power system. This FSC is installed in a relatively meshed part of the system where there are a number of long shafted generators, HVDC will soon be commissioned and an increasing number of wind turbines will be connected. This raises concerns over the possibility for:

1) SS resonance (SSR): series compensation interacting with generator torsional modes [15].

2) SS control interactions (SSCI): series compensation interacting with controllers [16].

3) SS torsional interactions (SSTI): power electronic converters interacting with generator torsional modes.

Extensive studies have been performed to verify that this FSC should not introduce any undesirable interactions. However, it is still of value to study the role that monitoring can play in alarming against any interactions and understanding the oscillations that exist in this range, which has not been previously studied using synchronized wide area measurements.

This range has not been studied using synchronized wide area measurements before because it is beyond the Nyquist limit for almost all PMUs $(25$ or $30 \mathrm{~Hz}$ ), as they report measurements once per cycle. VISOR is able to study this range because of the trial of the WMU (described in Sect. 3.1). An example of the oscillatory behavior observed in the GB system over a month is presented in Fig. 4.

It can be seen in Fig. 4 that the torsional modes are observed, albeit with varying rates of occurrence. Marked with an A in Fig. 4 is a mode with variable frequency, which is suggestive of control behavior. This is regularly observed at various locations and its occasional proximity to a torsional mode warrants further investigation. The detected modes will tend to be different depending on if the current or voltage is processed; this demonstrates the need to monitor both types of signal.

The detected oscillations are generally considered to be small and well-damped, with the majority in the region of $2 \mathrm{~V}$ and $0.1 \mathrm{~A}$ at the $400 \mathrm{kV}$ level.

\subsubsection{Source location for 0.002-4 Hz range}

The monitoring of the LF range is already part of day to day operation in GB and elsewhere [17, 18]. However, the 


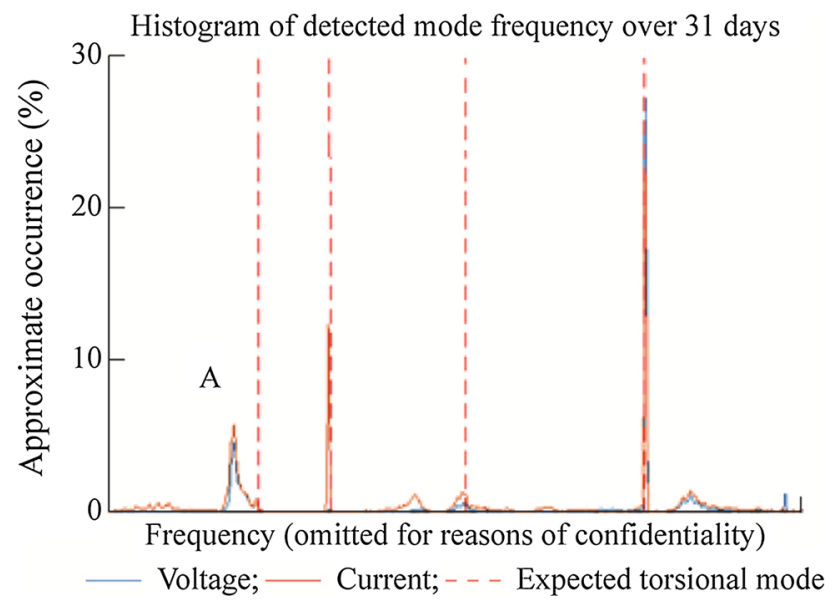

Fig. 4 Comparison of monitored frequency content and known modes

addition of source location to this existing monitoring would enable operators and planners to understand the source of any oscillations. This understanding could then be used as the basis for the development of tailored mitigating actions that target the specific source of the oscillation (e.g. a specific generator) and not just the symptoms of this oscillation (e.g. the power flow on a certain corridor). It is reasonable to expect that these tailored solutions will be more efficient than general solutions. This is attractive to the operator, as the management of oscillations will commonly involve limiting corridor flows and this will increase the cost of operation. Furthermore, especially in a market driven environment, it is attractive for those entities that are responsible for an operational issue to bear the responsibility for changing their behavior to prevent it.

Source location methods have been presented that use energy-based methods [19, 20]. These methods depend upon measurements of the power flow to trace oscillations to their source. However, power flow based tracing requires PMU observability of the power flows at the potential sources to function correctly, which limits their practical use in power systems with sparse PMU monitoring or for oscillations where the sources are unmonitored. Other methods use offline statistical analysis [21] of system data (e.g. dispatch, power flow measurements, estimated oscillation parameters) to identify correlations between oscillations and certain operating conditions, which may indicate a source location if a causal relationship can then be established through further investigation. These methods are well suited for source location with respect to oscillations that have repeat occurrences. However, by their nature, they are not suitable for online application or for dealing with oscillations that occur less frequently, due to a lack of sufficient data. This may be of concern for future systems that have far greater range of operating conditions and possible oscillatory interactions.
The source location method demonstrated within VISOR includes the novel use of voltage angles from PMUs. The source locations are identified using the relative phase of the measurements. A location with leading oscillation phase indicates a "source", i.e. it is reducing the damping of the mode, whilst a lagging phase indicates a "sink", i.e. it increases the mode damping.

In the case of opposing-phase oscillations, e.g. an interarea mode, the measurement locations are first separated into two coherent groups. The relative phase for each measurement is then calculated with respect to the average phase for its group. If the average phase of one group leads the average phase of the other group significantly then the most leading location within the leading group is considered to be the source of the oscillation. If neither group has a significant lead, then the leading members of each group are candidate sources.

The method has been applied to several offline study cases [22] and forms part of an online application in VISOR, by using phase angles the method is able to perform well in systems with only sparse measurements.

\subsection{Dynamic model validation}

Model based simulation of dynamic behavior is critical to the proper planning and operation of a power system. It is used for both steady state and post-fault contingency analyses to determine if the system is operating within security margins and quality of supply standards. Model inadequacies can thus have real and significant consequences for the power system. Overly conservative limits can lead to costly inefficient operation, whilst misleading stability assessment results can lead to separation or blackout, as in [23]. Validation and improvement of system models is therefore vital, and is likely to only become more challenging and resource intensive with the increasing complexity of power system plant, protection and control schemes and the range of possible operating conditions as we move toward a low carbon future.

WAMS data is ideally suited to model validation, being a continuous time-aligned record of steady-state and disturbed power system. It removes much of the effort and risk of error associated with collating other forms of data such as triggered fault records (which may be accurately timestamped but have different start/end times). Two main model validation approaches are raised in the literature [24-27]: system-wide and subsystem.

The system-wide approach uses simulations of the complete power system, and attempts to replicate the scenario under study by recreating it as a sequence of events (e.g. a line loss), validating the simulation results against WAMS data. This relies on accurate event reconstruction from records-in order to be sure that any observed 
discrepancies are due to the model rather than an unrepresentative simulation.

Subsystem model validation uses PMU measurements at the boundaries between a subsystem (e.g. generator) and the rest of the system to excite only the subsystem model. This effectively mimics the conditions the subsystem was exposed to during the scenario and validates the subsystem model using the PMU measurements recorded within it. A disadvantage of this approach, which has been encountered during VISOR, is that it is limited in application to subsystems that are fully bound by PMU measurements.

VISOR will assess the potential for validation of the GB system models using both of these approaches, with data from the VISOR WAMS. Furthermore, the extent to which SSO as well as small and large-signal behavior observed by VISOR is replicated in the system model will be assessed.

\subsection{Hybrid state estimation}

State estimation was one of the first applications proposed for synchronized phasor measurements [5]. Linear state estimation uses a fully observable set of PMU measurements to determine the system state using linear equations and a non-iterative procedure. However, this level of PMU monitoring is not feasible in the short to medium term for the GB system. Therefore, VISOR is focusing on the potential for HSE, where synchronized measurements from PMUs and non-synchronized measurements from remote terminal units (RTUs) are combined by the state estimator into a hybrid calculation [28]. This may allow the benefits of synchronized phasor measurements [29] to be realized without requiring a fully observable linear state estimator.

The focus of the VISOR HSE work is on improving the reliability of convergence, highlighted by the system operator to be of most immediate value. A literature review identified four main types of HSE: post processing, integrated, fusion and distributed. A post-processing HSE depends on the output of the existing state estimator and thus cannot improve convergence; fusion requires full PMU observability; and a distributed HSE uses local estimates rather than directly improving the central SE. Thus it was decided that VISOR would focus on the integrated HSE, which offers improved convergence by combining PMU data and SCADA data directly into a single, iterative estimation procedure. A post processing HSE will also be used as a means of comparison when assessing the accuracy improvement offered by the integrated HSE-which despite the focus on convergence should not be ignored.

The next stage of work will use offline simulations of IEEE benchmark systems to assess the improvements in convergence offered by the different types of integrated
HSE: rectangular current, pseudo flows, pseudo voltage, and constrained formulation.

\subsection{Line parameter estimation}

It has long been recognized that if phasor measurements are available at both ends of a transmission line then it will be possible to estimate the parameters of that transmission line. Improving the accuracy of the line parameters used when studying a power system could have real benefits to many applications, e.g. stability assessment.

However, in practice delivering this estimation with sufficient accuracy and robustness has proven challenging due to factors like poor measurement quality, variations in line construction and the inclusion of short cable runs in the transmission corridor [30-32]. For example, for a typical transmission line with an $X / R$ ratio of 10 , an angle error of $0.57^{\circ}$ (equal to the maximum $1 \%$ total vector error given in the C37.118.1 std) will result in a $10 \%$ error in the estimate of resistance.

The method that is being demonstrated in VISOR is a correlation based approach that has performed well when provided with data of sufficient quality. The example of the results achieved using measurements from PMUs in the GB system is shown in Fig. 5. Figure 5 shows 7 days of estimated resistance and reactance for a long line. Comparison of these estimated values with the nominal value shows that the method performs well in the presence of the combination of systematic and random errors that appear in actual power system measurements and are not considered in much of the existing work on LPE [30-32]. Systematic errors are a significant threat to LPE, as they will lead to fixed errors that may contribute to errors in decision making (e.g. when assessing a dynamic thermal line rating). Analysis of these results shows that the algorithm performs more poorly during periods of low load and that the algorithm has a bias toward overestimating the resistance, this bears further investigation.

However, the results obtained by VISOR, so far, for data from the GB power system have been limited by a shortage of lines that have PMUs at both ends and performance issues with the PMUs that are installed, e.g. angle drift and poor anti-aliasing.

\subsection{Laboratory testing of measurement devices}

Whilst not an application, the laboratory testing of the synchronized measurement devices that are in use within a WAMS is critical. This is because a proper understanding of the performance of these measurement devices is essential to ensuring that any applications deployed will perform reliably and correctly. Therefore, an ongoing aspect of VISOR will be the assessment of the performance 

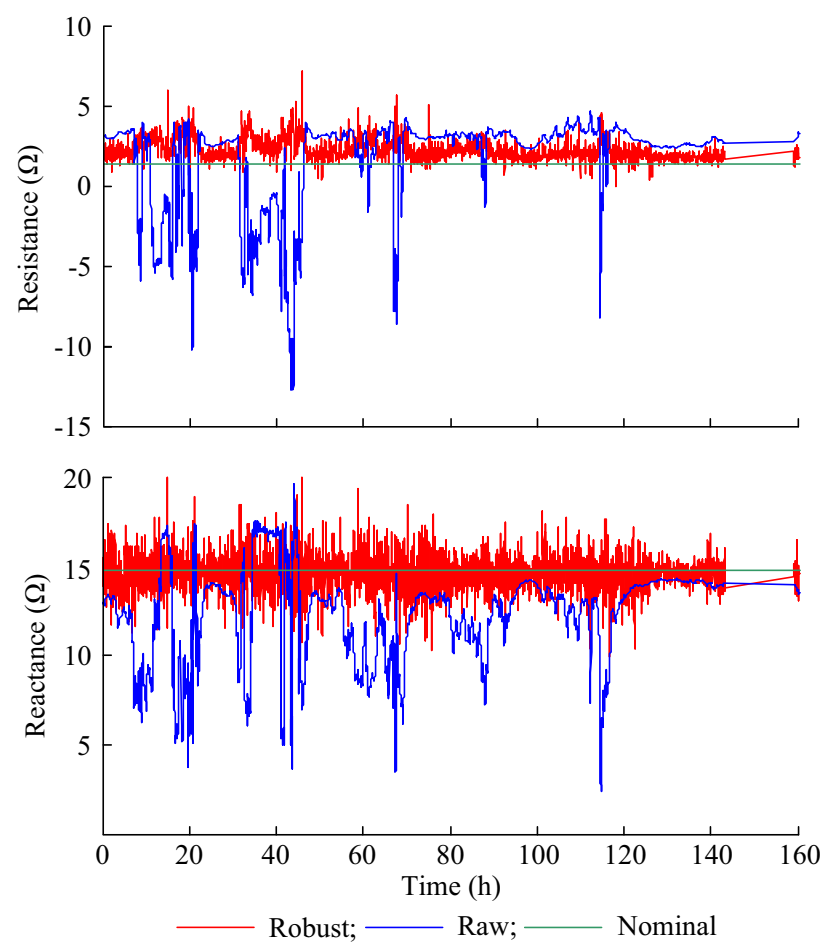

Fig. 5 Results from LPE trial in VISOR

of these measurement devices in a laboratory environment. This assessment will be tasked with identifying a baseline for the performance of these devices and will use the test setup presented in Fig. 6.

VISOR has performed a number of laboratory studies to understand the performance of SMT in the presence of subsynchronous oscillations.

One result of these studies is presented in Fig. 7. This result represents the response of a PMU to a sinusoidal voltage with fundamental frequency of $50 \mathrm{~Hz}$ and with six separate additive oscillation with frequencies of $1,5,10$, 20, 30 and $35 \mathrm{~Hz}$ and magnitude of 0.05 p.u. Each additive oscillation was sustained for 25 seconds with a 5 second gap between them to allow any transients to end before the next additive frequency was introduced. This example shows that when the additive oscillation is of a certain frequency it will destabilize the phasor estimation over

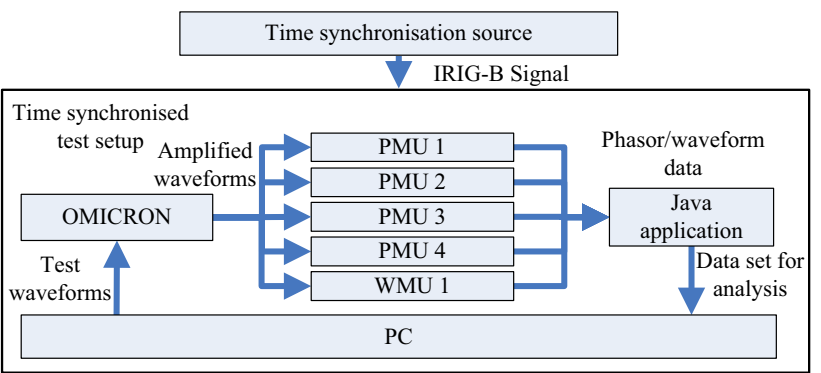

Fig. 6 Time synchronized test setup at The University of Manchester
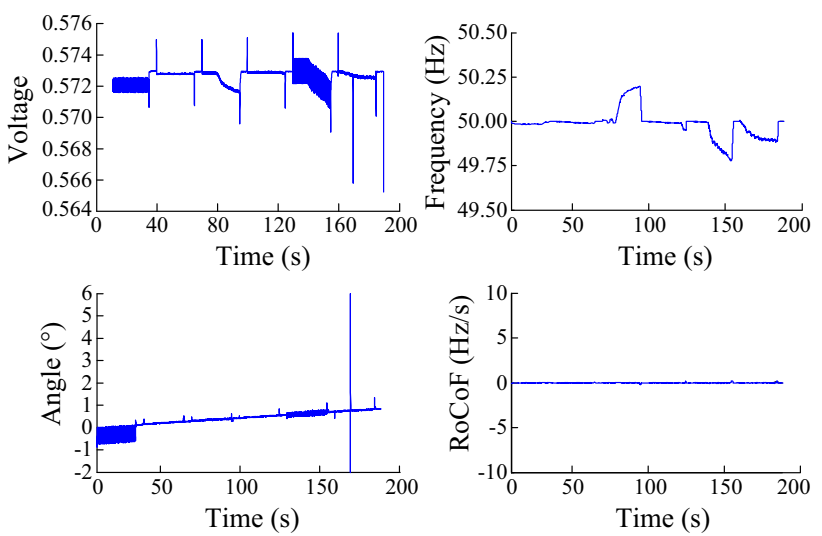

Fig. 7 PMU response to injection of a sequence of oscillations

time. For example, the response for a $10 \mathrm{~Hz}$ oscillation (the third block of injection in Fig. 7) is initially stable but over time the magnitude begins to fall and the frequency increases.

For comparison, the results of applying a fast Fourier transform (FFT) to the response of the WMU to these additive signals are presented in Fig. 8. The proper reporting of each frequency can be observed. However, the magnitude of each oscillation is not the same. Further analysis of these cases and other examples of the impact of subsynchronous oscillations on PMU performance can be found in [33].

Another example of the impact of subsynchronous oscillations on the performance of PMUs can be seen in the FFT analysis of the voltage magnitude reported by the four PMUS in Fig. 9. In this case the PMUs have been exposed to an additive $0.75 \mathrm{~Hz}$ oscillation.

PMU 2 and PMU 3 can be seen to wrongly report a 1.5 $\mathrm{Hz}$ oscillation for a $0.75 \mathrm{~Hz}$ injection. In contrast, the FFT output for PMU 1 and PMU 4 shows that, although the $0.75 \mathrm{~Hz}$ component is seen to have a distinctive spike, a

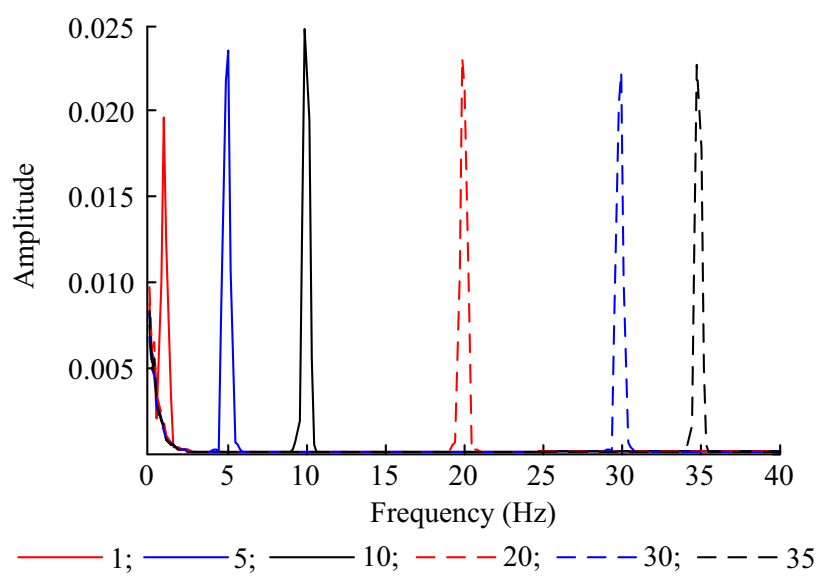

Fig. 8 FFT of WMU response to injection of a sequence of oscillations 

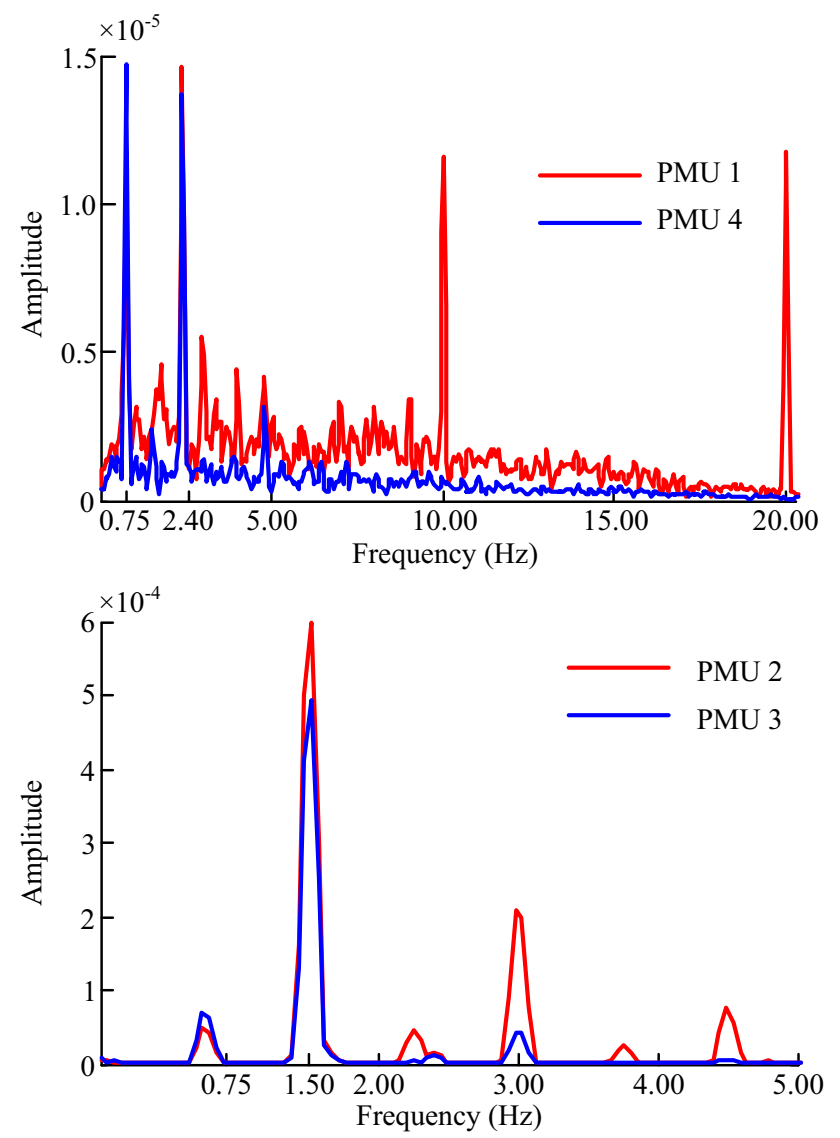

Fig. 9 Example of PMU response to a $0.75 \mathrm{~Hz}$ oscillation

$2.4 \mathrm{~Hz}$ component is reported with similar amplitude. Furthermore, PMU 1 reports similar amplitudes at 10 and $20 \mathrm{~Hz}$.

Further description of this laboratory set up, particularly the Java application developed, and examples of the results from this testing can be found in [34-37].

\section{Measurement device performance}

Delivering the VISOR WAMS is an ambitious undertaking, which requires the deployment of new sensors, communication links and computing hardware. However, deploying new assets is not the only challenge faced by VISOR. Integrating the existing WAMS assets in GB into the VISOR WAMS is an essential aspect of delivering the breadth of monitoring necessary at an acceptable cost. These assets were not used or maintained as part of a production system (i.e. they did not contribute to the everyday operation of the power system). Therefore, an essential aspect of VISOR is assessing the performance of these existing WAMS assets and incorporating them into the VISOR WAMS. Many of these are older devices, not initially designed for synchronized phasor measurement, which have subsequently received firmware updates to incorporate PMU functionality.

However, whilst this assessment is essential for incorporating the existing assets into the VISOR WAMS, it is important that this assessment is not limited to the preexisting WAMS assets. It must become an ongoing task that assesses the performance of new assets as they are deployed and verifies that the performance of the WAMS, or its component parts, does not degrade as the WAMS expands during the course of the project.

For the most part the existing assets that were to be incorporated into the VISOR WAMS were PMUs and some of the issues encountered during this assessment included: poor GPS reception, incorrect CT/VT wiring, and poor performance of communication links.

Issues of this nature are unsurprising, given that most of these assets were not used as part of a production system. However, certain issues were encountered that may be of more concern, as they are related to the measurement performance of the PMUs. These included the poor performance of internal clock oscillators, the unreported loss of time synchronism, the quantization of measurements and the intermittent failure to report measurements during stressed system conditions.

\subsection{Poor performance of internal crystal oscillators}

The angular difference across a transmission line, as reported by PMUs in the GB system is shown in Fig. 10. The saw tooth shape of the trace in this figure is caused by the poor performance of the crystal oscillator in one of the PMUs.

The PMU receives a one pulse per second signal from a GPS-locked time source. This is used as the basis for measurement synchronization and is particularly important as a reference for phase angle measurements. However, between these pulses the internal PMU clock relies on a crystal oscillator to maintain time and resets itself when the next pulse is received. In this case, the oscillator is not able to keep time correctly. Therefore, the PMU clock time and, consequently, the angle measured by the PMU will drift in the time between receiving the one pulse per second

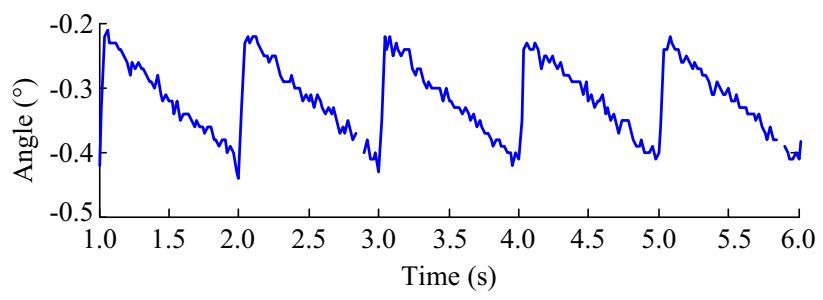

Fig. 10 Angular difference across a transmission line observed when internal crystal oscillator performs poorly 
signals. This can be seen in Fig. 10, as the saw tooth resets every second. This problem can be overcome by replacing the crystal oscillator in the PMU and a maintenance program to upgrade the affected PMUs has already begun.

\subsection{Unreported loss of synchronization}

The clear example of the output from a PMU that is not properly synchronized is shown in Fig. 11. This loss of synchronization went unreported by the WAMS. The C37.118 standard [12] requires PMUs to be able to detect and report the loss of their local synchronizing input. This is essential to the proper performance of a WAMS, as any error in the synchronization of the measurement devices will be interpreted as power system behavior (e.g. a larger or smaller angular separation) if it goes unreported.

The threat posed by the unreported loss of synchronization may appear limited, as cases of unreported loss of synchronization like those depicted in Fig. 11 appear trivial to identify visually. However, smaller errors that go unreported may not be as noticeable and may compromise the performance of WAMS applications, particularly those that depend on precise measurement of the relative angles across the system, e.g. LPE or oscillation source location.

\subsection{Quantization of frequency data}

The comparison of the frequency reported by four PMUs in the GB WAMS is shown in Fig. 12. From this it is clear that PMU 2 and PMU 4 quantize their reported frequency to a maximum precision of 0.00125 and $0.001 \mathrm{~Hz}$, respectively. The cause of this quantization appears to be different for each of the PMUs and in one case is due to the frequency calculation approach and hardware used in the PMU and in the other is due to the use of 16-bit integer format. The former issue can likely be overcome through a firmware upgrade of the device to allow the reporting of floating point numbers, although this will increase the bandwidth required by the PMU.

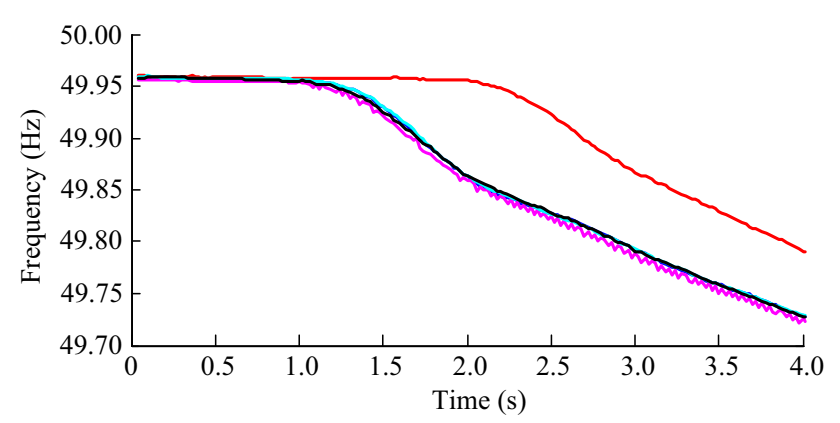

Fig. 11 Example of output of an unsynchronized PMU

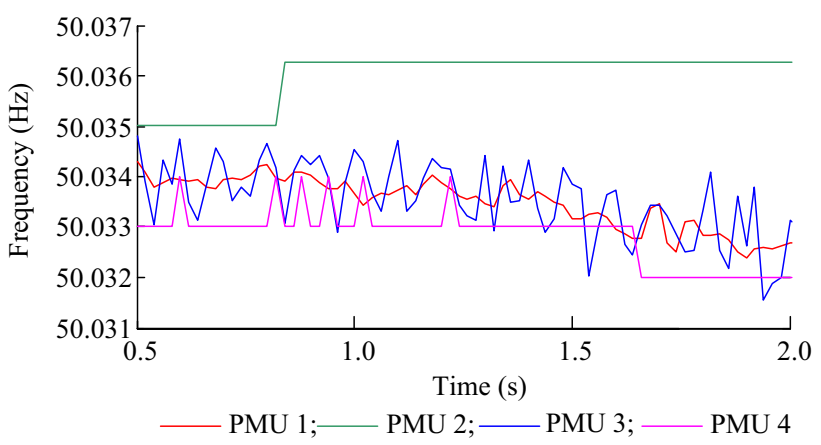

Fig. 12 Frequency reported by four PMUs (quantization is observable)

This quantization is within the $0.005 \mathrm{~Hz}$ accuracy required by the C37.118.1 standard [12] and in terms of monitoring large disturbances to the system frequency it is not a barrier to the proper performance of the WAMS. However, the quantization will prevent these PMUs from being used for the detection and characterization of the normal, low amplitude behavior of power system oscillations (e.g. inter-area oscillations) and from being used to localize the source of an oscillation.

\subsection{Quantization of current phasors}

The quantization of the current magnitude and angle reported by a PMU is shown in Figs. 13 and 14. This quantization is caused by a combination of the measurements being reported in rectangular form using 16-bit scales integers and the large full scale deviation used for the scaling of the current waveform. This large full scale deviation is used because the PMU is an upgraded fault recorder. Therefore, its primary role is the accurate measurement of full fault current and not the precise measurement of load current.

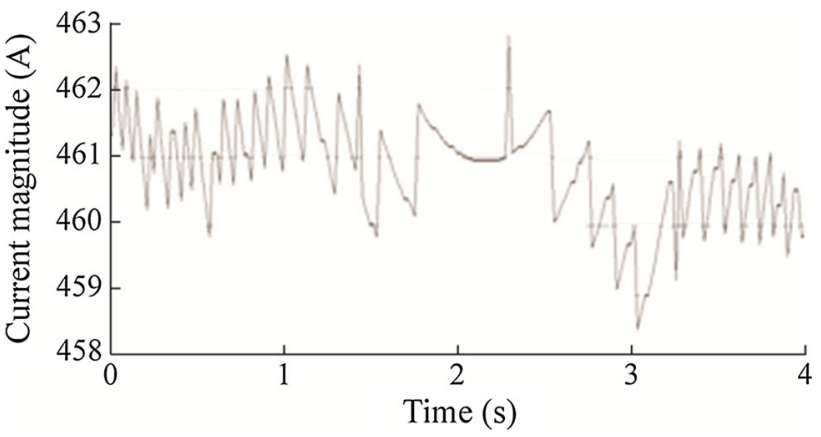

Fig. 13 Quantization of current magnitude measurements of PMU 


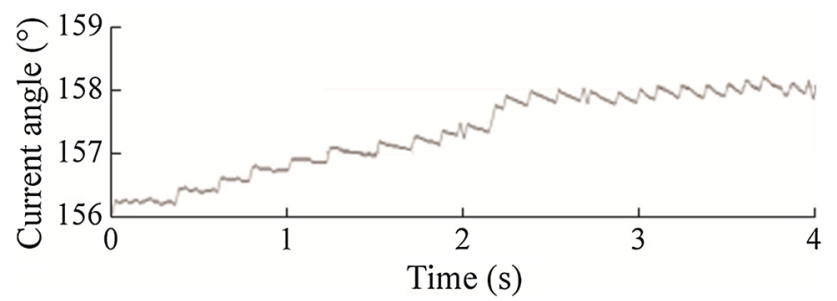

Fig. 14 Quantization of current angle measurements of PMU

\subsection{Intermittent failure to report measurements}

The example of an interesting phenomenon that has been observed in some of the PMUs installed in the GB WAMS is shown in Fig. 15. This example is a large disturbance in system frequency that occurred at approximately 10 seconds. The curve presented in red is the numerical frequency value reported by a PMU that is installed very close to the location of the disturbance. Two features are evident: the transient in the PMU output immediately after the disturbance and the rapid and false fluctuation in frequency that begins at approximately $25 \mathrm{~s}$ (shown in the inset of Fig. 15).

The first of these is due to the proximity of the PMU to the disturbance and is unsurprising. However, the second is of more interest. The inset in Fig. 12 shows that the PMU alternates intermittently between reporting $50 \mathrm{~Hz}$ and reporting seemingly accurate frequency measurements. The periods where $50 \mathrm{~Hz}$ values are reported are due to the PMU sending a default, "error" frame, which is correctly marked as invalid by the PMU. This issue is believed to be due to high processing load on the PMU, as a result of processing and storing a record of the disturbance. These error-tagged frames would be ignored by WAMS applications. However, the data lost during the disturbance is inconvenient, as this data is some of the data that would be of most interest to the user.

\subsection{Compatibility of monitoring devices with the required communication protocols}

The WMU being trialed as part of VISOR presently only streams data using the user datagram protocol (UDP) transport layer protocol, whilst the PMUs in the VISOR WAMS use transmission control protocol (TCP). This highlighted an important issue, as the use of UDP is not accepted as part of a production system in GB, due to the need for bi-directional opening of firewall ports.

For the purposes of an innovation level demonstration of the WMU and the applications it enables, as part of the VISOR project, this is not an obstacle. However, it must be addressed before any roll-out of the WMU based applications as part of a production system in GB. It should be noted that UDP is used in other WAMS worldwide, as it has reduced bandwidth requirements compared to TCP and enables the use of broadcast/multicast.

This situation is a good example of the value of innovation projects as part of the process for delivering the successful, efficient integration of new technologies into the day to day operation of power systems.

\section{Conclusion}

The VISOR project seeks to create the first WAMS to monitor the entire GB power system and based on this showcase the benefits of WAMS to the GB system. The ultimate goal of the VISOR project is to help build the case

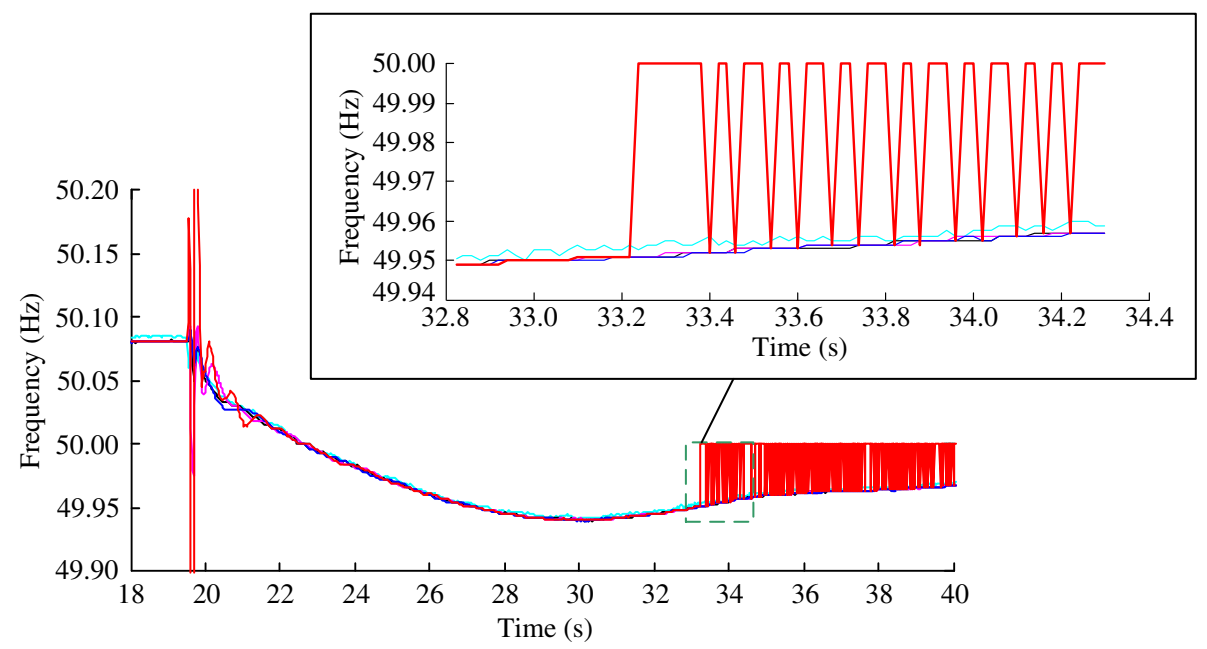

Fig. 15 PMU sending invalid packets after a major disturbance 
for business as usual deployment of WAMS in GB. The paper details some of the WAMS applications that are being developed within VISOR and presents some of the results to date.

This paper also presents some of the issues that have been encountered during the deployment and ongoing review of the WAMS assets in GB. These issues are not unexpected, given the non-production nature of the WAMS in GB. However, they are indicative of the issues that will be encountered during the staged deployment of WAMS. A staged deployment is inevitable, due to the scale of a WAMS and the scale of capital and operational expenditure required to deliver a suitable WAMS

Acknowledgment This work was supported by the GB Network Innovation Competition (NIC).

Open Access This article is distributed under the terms of the Creative Commons Attribution 4.0 International License (http:// creativecommons.org/licenses/by/4.0/), which permits unrestricted use, distribution, and reproduction in any medium, provided you give appropriate credit to the original author(s) and the source, provide a link to the Creative Commons license, and indicate if changes were made.

\section{References}

[1] Terzija V, Valerde G, Cai DY et al (2011) Wide-area monitoring, protection, and control of future electric power networks. P IEEE 99(1):80-93

[2] Chakrabarti S, Kyriakides E, Bi TS et al (2009) Measurements get together. IEEE Power Energ Mag 7(1):41-49

[3] US-Canada Power System Outage Task Force (2004) Blackout 2003: final report on the August 14, 2003 blackout in the United States and Canada: causes and recommendations. Office of Electricity Delivery \& Energy Reliability, Washington

[4] Report of the Enquiry Committee on grid disturbance in northern region on 30th July 2012 and in northern, eastern \& northeastern region on 31st July 2012. The Enquiry Committee, Ministry of Commerce and Industry, Government of India, New Delhi, India, 2012

[5] Phadke AG, Thorp JS (2008) Synchronized phasor measurements and their applications. Springer, Boston

[6] Regulator of GB electricity markets. The Office of Gas and Electricity Markets (OFGEM), London, UK, 2002

[7] Electricity network innovation competition full submission proforma-Section 1: Project summary. Visualisation of Real Time System Dynamics using Enhanced Monitoring (VISOR) project

[8] NASPI 2014 survey of synchrophasor system networks-results and findings. North American SynchroPhasor Initiative (NASPI), 2015

[9] Electricity ten year statement 2015. National Grid, London, 2015

[10] System operability framework 2015. National Grid, London, 2015

[11] NETS Security and quality of supply standard. National Grid, London, 2014

[12] IEEE Std C37.118.2-2011. IEEE standard for synchrophasor data transfer for power systems. 2011
[13] Novosel D (2007) Phasor measurement application study. Final Project Report, California Institute for Energy and Environment (CIEE), University of California, Berkeley

[14] Cai DY, Wall P, Osborne M et al (2016) Roadmap for the deployment of WAMPAC in the future GB power system. IET Gener Transm Distrib 10(7):1553-1562

[15] Padiyar KR (1998) Analysis of subsynchronous resonance in power systems. Kluwer Academic Publishers, Boston

[16] Gross LC (2010) Sub-synchronous grid conditions: new event, new problem, and new solutions. In: Proceedings of the 37th annual western protective relay conference, Spokane, 19-21 Oct 2010, $19 \mathrm{pp}$

[17] Identification of electromechanical modes in power systems. TP462, IEEE Power and Energy Society, Piscataway, 2013

[18] Cai DY, Regulski P, Osborne M et al (2013) Wide area interarea oscillation monitoring using fast nonlinear estimation algorithm. IEEE Trans Smart Grid 4(3):1721-1731

[19] Chen L, Min Y, Hu W (2012) An energy-based method for location of power system oscillation source. IEEE Trans Power Syst 28(2):828-836

[20] Yu YP, Min Y, Chen L, et al (2011) The disturbance source identification of forced power oscillation caused by continuous cyclical load. In: Proceedings of the 4th international conference on electric utility deregulation and restructuring and power technologies (DRPT'11), Weihai, 6-9 Jul 2011, pp 308-313

[21] McNabb P, Wilson D, Bialek J (2013) Classification of mode damping and amplitude in power systems using synchrophasor measurements and classification trees. IEEE Trans Power Syst 28(2):1988-1996

[22] Al-Ashwal N, Wilson D, Parashar M (2014) Identifying sources of oscillations using wide area measurements. In: Proceedings of the CIGRE US National Committee 2014 grid of the future symposium, Houston, 19 Oct 2014

[23] Allen E, Kosterev D, Pourbeik P (2010) Validation of power system models. In: Proceedings of the 2010 IEEE Power Engineering Society general meeting, Minneapolis, 25-29 Jul 2010, $7 \mathrm{pp}$

[24] Huang ZY, Yang B, Kosterev D (2009) Benchmarking of planning models using recorded dynamics. In: Proceedings of the 2009 IEEE Power and Energy Society power systems conference and exposition, Seattle, 15-18 Mar 2009, 6 pp

[25] Ma J, Han D, Sheng WJ et al (2008) Wide area measurementsbased model validation and its application. IET Gener Transm Distrib 2(6):906-916

[26] Huang ZY, Kosterev M, Guttromson R, et al (2006) Model validation with hybrid dynamic simulation. In: Proceedings of the 2006 IEEE Power Engineering Society general meeting, Montreal, 18-22 Jun 2006, 9 pp

[27] Ghiocel SG, Chow JH, Stefopoulos G et al (2014) Phasormeasurement-based state estimation for synchrophasor data quality improvement and power transfer interface monitoring. IEEE Trans Power Syst 29(2):881-888

[28] Valverde G, Chakrabarti S, Kyriakides E et al (2011) A constrained formulation for hybrid state estimation. IEEE Trans Power Syst 26(3):1102-1109

[29] Phadke AG, Thorp JS, Karimi KJ (1986) State estimation with phasor measurements. IEEE Power Eng Rev 6(2):48

[30] Wilson RE, Zevenbergen GA, Mah DL et al (1999) Calculation of transmission line parameters from synchronized measurements. Electr Mach Power Syst 27(12):1269-1278

[31] Liao Y, Kezunovic M (2009) Online optimal transmission line parameter estimation for relaying applications. IEEE Trans Power Delivery 24(1):96-102

[32] Shi D, Tylavsky DJ, Logic N, et al (2008) Identification of short transmission-line parameters from synchrophasor 
measurements. In: Proceedings of the 40th North American power symposium (NAPS '08), Calgary, 28-30 Sept 2008, 8 pp

[33] Wall P, Dattaray P, Nechifor A, et al (2015) Monitoring subsynchronous oscillations in power systems using synchronised measurement technology. In: Proceedings of the CIGRE Study Committee B5 (protection and automation) colloquium, Nanjing, 23-24 Sept 2015

[34] Wall P, Dattaray P, Mohapatra P, et al (2015) VISOR project: opportunities for enhanced real time monitoring and visualization of system dynamics in GB. In: Proceedings of the 6th annual protection automation and control world conference, Glasgow, 29 Jun-2 Jul 2015, 23 pp

[35] Nechifor A, Albu M, Hair R, et al (2015) Development of a WAMS laboratory for assessing PDC compliance with the IEEE C37.244 standard. In: Proceedings of the 6th international conference on advanced power system automation and protection (APAP'15), Nanjing, 21-23 Sept 2015

[36] Regulski P, Wall P, Rusidovic Z, et al (2014) Estimation of load model parameters from PMU measurements. In: Proceedings of the 2014 IEEE PES innovative smart grid technologies conference, Europe (ISGT-Europe'14), Istanbul, 12-15 Oct 2014, 6 pp

[37] Wall P, Regulski P, Rusidovic Z, et al (2014) Inertia estimation using PMUs in a laboratory. In: Proceedings of the 2014 IEEE PES innovative smart grid technologies conference, Europe (ISGT-Europe'14), Istanbul, 12-15 Oct 2014, 6 pp

Peter WALL graduated from the University of Manchester with a Bachelors degree in Electrical and Electronic Engineering (2008), a Masters degree in Power Systems (2009) and a Ph.D. in Power Systems (2013). His main area of interest is wide area monitoring, frequency stability and intelligent controlled islanding. He is currently a post-doctoral research associate at The University of Manchester.

Papiya DATTARAY received her Bachelor degree from the West Bengal University of Technology in Electrical Engineering followed by a Masters in Power system engineering from the Indian Institute of Technology Delhi (IITD) in 2010 and 2012 respectively. She then worked at the GE Global Research Centre, JFWTC, Bangalore, India for a year before serving as a research associate at TU Dresden for 6 months. Since 2014 she has been pursuing her Ph.D. degree at The University of Manchester and her key research focus is subsynchronous resonance and torsional interactions, power system dynamics and control.

Zhaoyang JIN received the Bachelor degree in Electrical and Electronic Engineering from the University of Manchester, Manchester, UK and North China Electric Power University, Baoding, China in 2013. Since January 2014 he has been pursuing a Ph.D. degree at The University of Manchester. His main research interests include wide area monitoring, static and dynamic state estimation.

Priyanka MOHAPATRA is currently working as Senior Project Manager within the Future Networks department at SP Energy Networks. She is leading two Network Innovation Competition projects, VISOR and FITNESS, and is also involved in conceptualization of future NIC proposals. Prior to joining SPEN; Priyanka was working with Siemens AG for 8 years. She started at Siemens Ltd India as an electrical engineer. She then moved on to Siemens AG Global R\&D, Germany working as a software developer and project manager for SCADA, EMS, DMS systems. She then worked with Siemens Protection Devices Ltd., UK as a product owner and senior engineer designing and developing protection devices. Priyanka holds a B.Tech. in Electrical Engineering and a M.Sc. in Renewable Energy
Systems Technology, and is enthusiastic about engineering and environment.

James YU is Future Networks manager for Scottish Power Energy Networks in the UK. In this role he is responsible for drafting and delivering the companies innovation strategy. James received and M.Sc. in Electrical and Electronic Engineering from the University of Newcastle-Upon-Tyne in 2000 and a Ph.D. from Northumbria University in 2003.

Douglas WILSON is Chief Technology Officer with GE Grid Solutions. He is responsible for development of applications and solutions. He also works with customers to analyze and resolve various problems observed through WAMS, and in areas such as generator testing, model validation and PSS tuning. He has participated in and led various collaborative $R \& D$ projects with industrial and academic partners.

Karine HAY is a senior application engineer at GE Grid Solutions. She is involved in the research and development of new real-time WAMS software applications and in delivering consultancy to power system utilities. She graduated from the University of Rennes 1 with an M.Sc. degree in Telecommunications (1994) and Ph.D. in Signal Processing (1997).

Stuart CLARK received his M.Eng. degree in Electrical \& Electronic Engineering from the University of Bristol in 2012. He has been involved in power system monitoring and in particular Wide Area Monitoring Systems (WAMS) for 5 years-specializing in research \& development, consulting and delivery concerning WAMS applications and infrastructure. Stuart is currently a Senior Power Systems engineer at the GE Grid Solutions WAMS Centre of Excellence in Edinburgh, UK.

Mark OSBORNE graduated from Lancaster University with a Bachelor degree in Physics (1991), Master degree in Mechatronics (1992) and Ph.D. in Electromagnetic Transient modelling (1997). His areas of responsibility include substation technology integration, wide area monitoring and insulation coordination. $\mathrm{He}$ is a technology application Engineer at National Grid.

Phillip M. ASHTON received the M.Eng. degree from the University of Portsmouth, Portsmouth, UK, in 2006 and an Engineering Doctorate (Eng.D.) degree from Brunel University, London, UK, based in industry with the electricity transmission system operator, National Grid. His current research interests include exploiting the use of phasor measurement units for enhanced operation and control of the GB transmission system.

Vladimir TERZIJA was born in Donji Baraci (former Yugoslavia). He received the Dipl-Ing., M.Sc., and Ph.D. degrees in electrical engineering from the University of Belgrade, Serbia, in 1988, 1993, and 1997, respectively. He is the Engineering and Physical Science Research Council Chair Professor in Power System Engineering with the School of Electrical and Electronic Engineering, The University of Manchester, UK. He was an Assistant Professor at the University of Belgrade, Serbia before becoming a senior specialist for switchgear and distribution automation with ABB AG Inc., Ratingen, Germany. His current research interests include wide-area monitoring, protection, and control; switchgear and fast transient processes; and digital signal processing applications in power systems. Prof. Terzija is Editor in Chief of the International Journal of Electrical Power and Energy Systems, an Alexander von Humboldt Fellow, as well as a DAAD and Taishan Scholar. 